•综述・

\title{
桑寄生植物繁殖物候研究概述
}

\author{
李慢如 ${ }^{1,2}$ 张 玲 ${ }^{*}$ \\ 1 (中国科学院西双版纳热带植物园热带森林生态学重点实验室, 云南预腊 666303) \\ 2 (中国科学院大学, 北京 100049)
}

\begin{abstract}
摘要: 桑寄生植物是一类自身可以进行光合作用的半寄生性灌木, 作为森林和林地的关键性资源可为鸟类等动物 分类群提供重要的食物资源和巢址, 并影响当地的生物多样性。桑寄生植物隶属于檀香目, 包括5科88属约 1,600 种, 除极地、部分高寒和干旱沙漠地区外均有分布。桑寄生植物繁殖物候的研究对于了解“寄主植物-桑寄生植物传粉/种子散布者系统” 中物种相互作用网络的维持机制、生态系统结构的稳定性具有重要意义。本文综述了桑寄 生植物在景观、群落和种群尺度上繁殖物候的表现类型, 发现桑寄生植物主要通过繁殖物候异步的方式延长物候 期, 维持与传粉/种子散布者持久的互惠关系以保障自身种群的繁衍。该领域经过近年的发展, 已从单一地描述繁 殖物候的表现类型到探究系统中寄主植物、传粉/种子散布者以及桑寄生植物自身生物学特性等因素的分析。通过 前人的研究发现桑寄生植物繁殖物候对于该系统内相互作用的双方或多方有重要的适应意义, 今后首先应该对桑 寄生植物的基础生物学背景进行研究, 然后还应加强实验验证, 进行多因素综合分析等来探讨桑寄生植物繁殖物 候的生态学意义。
\end{abstract}

关键词: 桑寄生; 繁殖物候; 寄主植物; 传粉者; 种子散布者

\section{Overview of the reproductive phenology of mistletoes}

\author{
Manru $\mathrm{Li}^{1,2}$, Ling Zhang ${ }^{{ }^{*}}$ \\ 1 CAS Key Laboratory of Tropical Forest Ecology, Xishuangbanna Tropical Botanical Garden, Chinese Academy of \\ Sciences, Mengla, Yunnan 666303 \\ 2 University of Chinese Academy of Sciences, Beijing 100049
}

\begin{abstract}
Mistletoes are a polyphyletic group of hemi-parasitic shrubs that can conduct photosynthesis. As keystone resources, they provide important food resources and nest sites for many birds and other fauna in forests and woodlands, mediating local biodiversity. This group is comprised of 5 families, 88 genera and about 1,600 species within the Santalales and is broadly distributed across terrestrial ecosystems, absent only in some alpine, desert and polar areas. Research into the reproductive phenology of mistletoes is important for understanding the maintenance of species interaction networks in "host-mistletoe-pollinator/seed disperser" systems and the stability of ecosystem structure. We review the reproductive phenology of mistletoes at landscape, community, and population scales. We begin by describing different forms of mistletoe reproductive phenology, and continue by exploring the evolutionary and developmental drivers of host species, pollinator/seed disperser, and mistletoe biological characteristics. These studies indicate that long-lasting mutualisms between pollinators/seed dispersers and mistletoes are maintained mainly by asynchronous phenology of mistletoes in order to ensure their reproductive success. Previous studies found that the reproductive phenology of mistletoes had important adaptive significance to interacting species in "host-mistletoe-pollinator/seed disperser" systems. Future efforts should first prioritize basic biological research on mistletoes to fill important data gaps, and then couple experimental verification with comprehensive multi-factor analysis to strengthen our understanding of the ecological significance of mistletoe reproductive phenology.
\end{abstract}

Key words: mistletoes; reproductive phenology; host plants; pollinator; seed disperser

收稿日期: 2019-11-06; 接受日期: 2020-01-25

* 通讯作者 Author for correspondence. E-mail: zhangl@xtbg.org.cn 
植物繁殖时间的安排和组合构成了植物具有 季节性和周期性的繁殖物候(reproductive phenology) (Elzinga et al, 2007)。植物的繁殖物候是植物生活史 的重要特征, 不仅影响植物自身的传粉、种子散布 和后代质量(Sun \& Frelich, 2011; Craine et al, 2012), 同时也影响依赖于这些植物获取资源的动物 (Newstrom et al, 1994; Wright \& Meagher, 2003)。通 常情况下植物的繁殖物候可以分为开花物候和结 果物候, 通过对植物始花期、盛花期、花期/果期长 短、个体/种群的同步性指数等多个物候参数的量化 可以详细地描述植物的繁殖物候(SanMartin-Gajardo \& Morellato, 2003)。其中繁殖物候同步(reproductive phenological synchrony)是指植物个体间或个体内水 平上花期或果期的重叠现象, 重叠程度使用同步性 指数(0-1)表示, 数值越大表示同步性程度越高, 异 步性程度越低(Bolmgren, 1998)。与此相反, 繁殖物 候异步是用来描述物候事件开始时间或持续时长 的错开程度, 使用数值1减去同步性指数的差值可 用来表示异步性程度(Augspurger, 1983; Elzinga et al, 2007)。繁殖物候异步对于植物来说有诸多好处, 如改变花及果实资源的时空分配(Quintana-Rodriguez et al, 2018), 在天气或资源条件不确定的情况下降 低传粉不确定性的风险(Rathcke \& Lacey, 1985)、逃 避种子捕食者(Pettersson, 1994), 以及实现果实的 不同步成熟，从而增加种子被成功散布的可能性 (Thompson \& Willson, 1979; Gorchov, 1988)。

桑寄生植物(mistletoes)是一类自身可以进行光 合作用的半寄生性灌木, 它们分属于檀香目下的桑 寄生科、檞寄生科、羽毛果科、榄仁檀科和檀香科 (部分)的 88 个属, 近 1,600 种植物, 除极地、部分高寒 和干旱沙漠地区外均有分布(Watson，2011) (图1), 其中桑寄生科和檞寄生科的物种多样性最高 (Nickrent, 2011; Liu et al, 2018)。大部分桑寄生植物 都能够产生丰富的花蜜和大量颜色鲜艳的果实, 为 许多传粉和种子散布者提供丰富的食物资源 (Watson \& Herring, 2012), 由于它们半寄生的特性, 绝大多数桑寄生植物都需要依靠鸟类或哺乳动物 进行种子散布才有可能到达合适的寄主植物完成 定殖(Amico \& Aizen, 2000; Watson, 2001; Mathiasen et al, 2008)。达尔文也曾将桑寄生植物与动物之间 的相互作用关系视为进化适应的早期范例 (Burkhardt \& Smith, 1990), 但由于桑寄生植物与传
粉/种子散布者之间的关系没有“榕-传粉榕小蜂系 统” 中互惠双方那么专性的相互作用, 因此被认为 是一种弥散型协同进化(diffuse coevolution) (Reid, 1991; Nickrent, 2002; Watson \& Herring, 2012), 加 之与寄主植物的广泛连接, 也形成了 “寄主植物-桑 寄生植物-传粉/种子散布者系统” (Mathiasen et al, 2008)。

桑寄生植物在森林和林地系统扮演着关键种 (keystone species)和关键性资源(keystone resources) 的角色(Watson, 2001; Press \& Phoenix, 2005; Watson \& Herring, 2012)。早在1983年, 印度的一项研究就 表明同域分布的多种桑寄生植物繁殖物候在不同 地点存在异步现象, 其可以在景观尺度上延长为传 粉者以及种子散布者提供食物资源的时长，还第一 次提出了“桑寄生植物繁殖物候的异步是为了维持 与传粉者和种子散布者的互利共生关系”这一生态 学观点(Davidar, 1983b)。后来澳大利亚生态学家 Watson在2001年发表的综述文章中也指出桑寄生 植物繁殖物候的特殊性对于维持“寄主植物-桑寄 生植物一传粉/种子散布者系统”的持久性和稳定性 具有重要作用(Watson, 2001)。然而目前支持这一观 点的案例还较少, 影响桑寄生植物繁殖物候的因素 与寄主植物和传粉者/种子散布者的作用关系也有 所差异, 并且有关这一观点的生态学意义的分析还 较为笼统, 国内研究案例更加贵乏。本文以国外相 关研究文献为主, 总结不同研究尺度下桑寄生植物 的繁殖物候表现类型，分析影响繁殖物候的因素， 概述它们在与传粉/种子散布者和寄主植物相互作 用中所发挥的重要适应意义, 并对未来的研究进行 展望。

\section{桑寄生植物繁殖物候的研究尺度及表现}

已有的研究发现桑寄生植物有两种主要的开 花方式(多表现在桑寄生科): 一种是不需要依靠外 力作用便可正常开放(Ladley \& Kelly, 1995; Ladley et al，1997); 另一种是 “爆炸性”开花，即当传粉者 拜访时接触到花冠管顶部, 花朵突然开放(Feehan, 1985; Ladley \& Kelly, 1995)。除了像桑寄生科的 Dendrophthoe falcata 等植物花果期同步程度较高外 (Davidar, 1983b), 桑寄生植物还可通过两种方式实 现繁殖物候的异步，即同种桑寄生植物的不同个体 

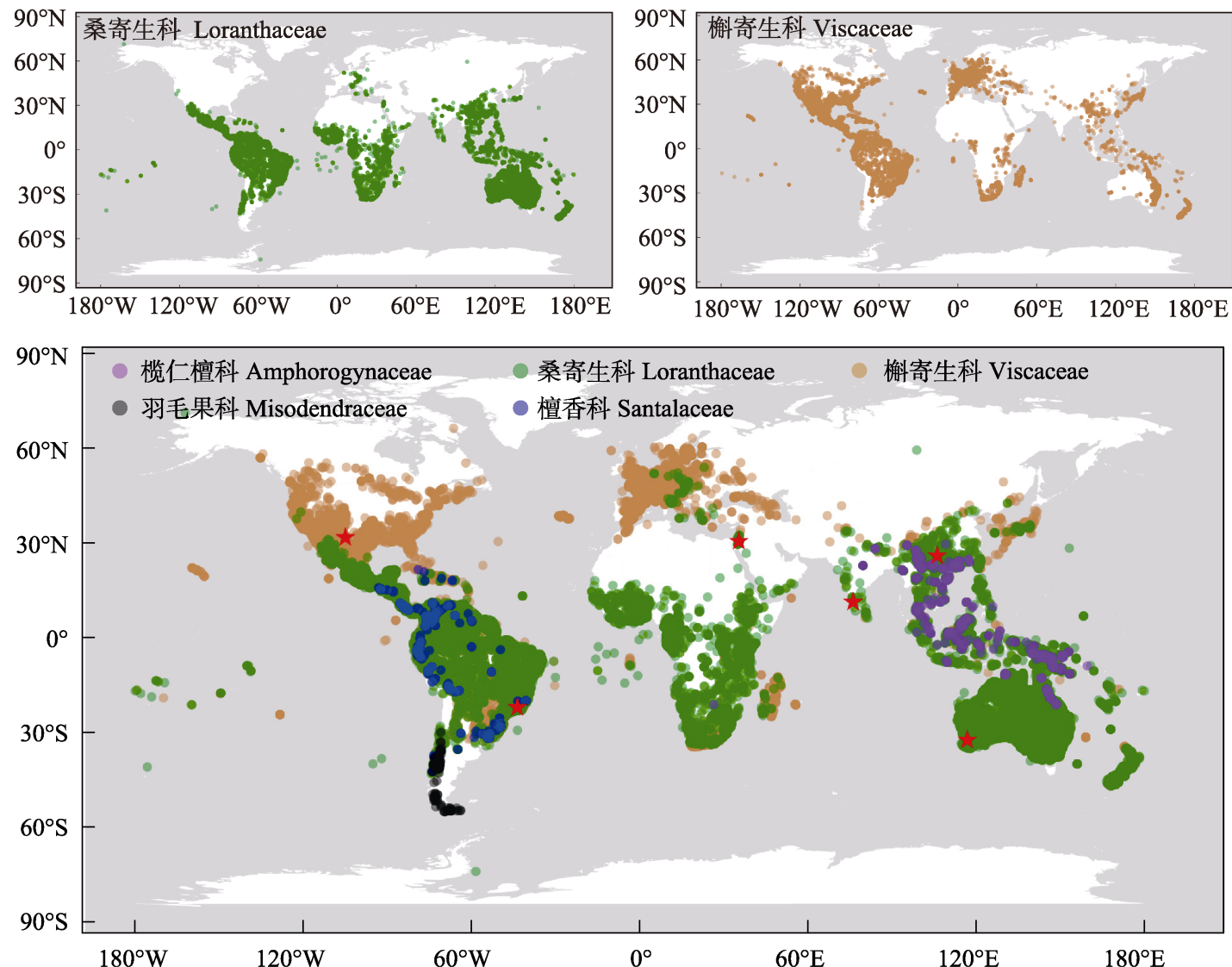

图1 桑寄生植物分布地图(红色星形表示文献中桑寄生植物繁殖物候表现类型的研究地点)。使用RStudio软件maptools和 dismo程序包在线获取全球生物多样性信息网络数据库Global Biodiversity Information Facility (GBIF) (https://ww.gbif.org/) 桑寄生植物属一级的经纬度数据绘制。

Fig. 1 The distribution map (latitude and longitude data) of genera of mistletoes from the Global Biodiversity Information Facility (GBIF) (https://ww.gbif.org/) is drawn by using maptols and dismo packages in R Studio software.

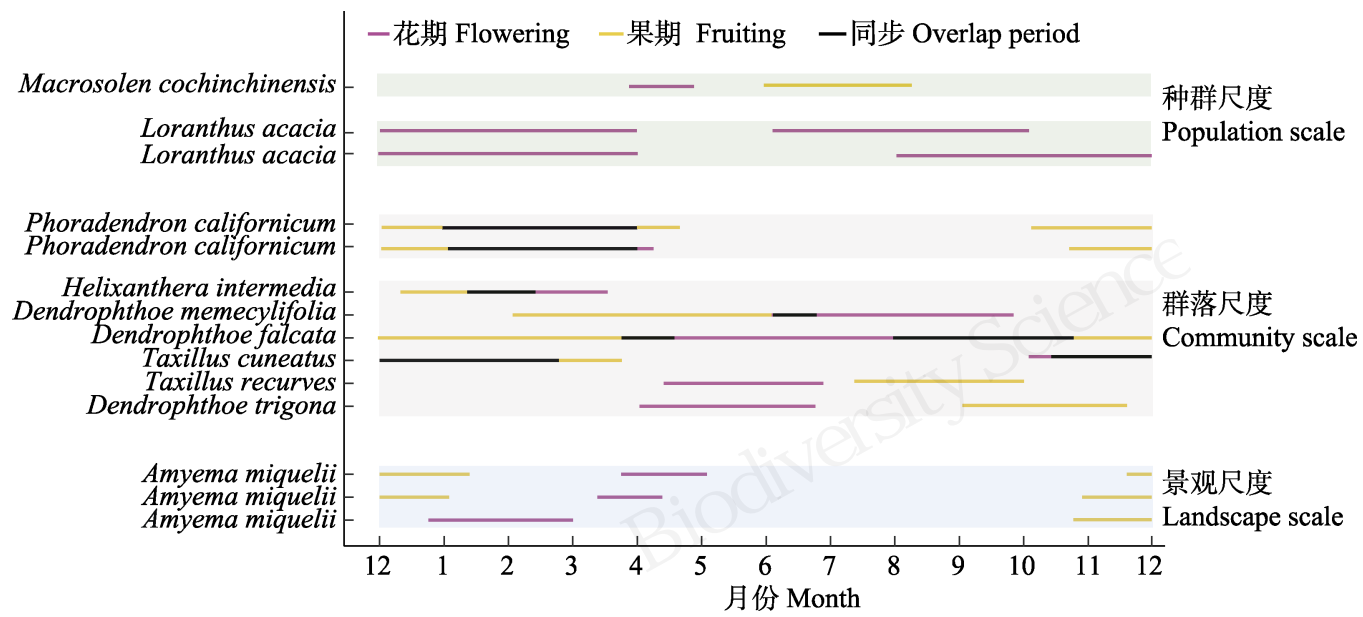

图2 桑寄生植物繁殖物候在不同研究尺度上的表现类型

Fig. 2 Reproductive phenology types of mistletoes at different study scales 
开花和果实成熟的不连续以及种群间/内植物个体 间花果期的时间错开或持续时长不同 (Davidar, 1983b; Watson, 2001), 从而延长单个物种或群落的 花果期, 以保证取食花蜜和果实的鸟类或哺乳动物 在全年都能获得可利用的食物资源(Davidar, 1983a; Mathiasen et al, 2008)。结合研究观测尺度的大小, 桑寄生植物的繁殖物候类型可体现为以下 3 个尺度 (图 2): (1)景观尺度: 同种桑寄生植物 Amyema miquelii 在不同地点花果期开始时间和持续时长不 同(Napier et al, 2014)。(2)群落尺度：同一群落内不 同桑寄生植物种类 Dendrophthoe trigona, Taxillus recurves, Taxillus cuneatus, Dendrophthoe falcata, Dendrophthoe memecylifolia, Helixanthera intermedia 的花果期彼此错开又有部分重叠(Davidar，1983b); 同一群落内不同寄主植物上同种桑寄生植物 Phoradendron californicum 的花果期不同(Yule \& Bronstein, 2018b)。(3)种群尺度：同种桑寄生植物 Loranthus acacia 的不同个体具有不同的开花物候 模式 (Vaknin et al, 1996); 同种桑寄生植物 Macrosolen cochinchinensis 的花期与果熟期之间彼 此错开(李慢如和张玲, 2019)。

\section{桑寄生植物繁殖物侯的影响因素}

桑寄生植物处在“寄主植物-桑寄生植物-传粉/ 种子散布者系统”之中, 它们的繁殖物候除了受到 环境因子的影响之外, 还会受到寄主植物、传粉者/ 种子散布者和自身生物学特性的影响。

\section{1 寄主植物的影响}

(1)寄主植物种类的影响：已有研究表明大多 数桑寄生植物能同时感染不同种类的寄主植物 (Press \& Phoenix, 2005; Shen et al, 2006), 最著名的 例子就是檞寄生科的白果槲寄生(Viscum album), 仅在欧洲地区感染的寄主就达450多种(Barney et al, 1998); 桑寄生科的五芯寄生 (Dendrophthoe pentandra) 在中国的西双版纳地区也可在 200 多种 植物上寄生(王煊妮和张玲, 2017)。因与寄主植物广 泛连接，它们的繁殖物候可能会受寄主植物种类的 影响(Teixeira-Costa et al, 2017; Twyford, 2018)。 Overton (1997)对生长在索诺兰沙漠的檞寄生科雌 雄异株植物Phoradendron californicum的繁殖物候 进行研究, 结果表明感染在同域分布的不同寄主植 物种类上的 $P$. californicum盛花期相差约 42 天，后
来采用现代分子生物学方法证明了不同寄主种类 上的P. californicum已经发生了明显的遗传分化 (Yule et al, 2016), 而它的果期物候却没有受到寄主 种类的影响(Yule \& Bronstein, 2018b)。以上研究表 明了寄主植物种类在介导桑寄生植物高度可塑的 繁殖物候中可能发挥了重要作用。但对桑寄生科 Loranthus acacia植物花期的研究却发现寄主种类 没有显著的影响作用(Vaknin et al, 1996)。相似的结 果也在澳洲西南部桑寄生科植物Amyema preissii上 得到验证(Napier et al, 2014)。除了桑寄生植物本身 存在的生物学特性差异会影响研究结果外, 样本量 大小也会影响物候曲线的拟合，从而使得桑寄生植 物或者寄主植物的繁殖物候格局表现不同(Clark \& Thompson, 2011)。

(2)寄主植物物候的影响：桑寄生植物通过与 其寄主植物的木质部相连从而获取水分、矿物质和 养分(Aukema, 2003; Shen et al, 2006)，当寄主植物 积累一定的物质和能量去完成自身某一生活史事 件时, 将会在一定程度上影响桑寄生植物的能量积 累(Bannister \& Strong, 2001; Wang et al, 2008)。 Godschalk (1983)对南非Loskop Dam自然保护区的 两种桑寄生科植物 (Tapinanthus natalitius 和 $T$. leendertziae)的研究发现, 随着落叶性寄主植物越 冬之后生理活动变得活跃, 其上的桑寄生植物也进 入了自身的繁殖周期，而经过传粉之后发育进程便 慢了下来。对西双版纳地区桑寄生科鞘花 (Macrosolen cochinchinensis)的研究也发现, 其始花 期与寄主植物木荷(Schima superba)的始花期相关 性较高, 两种植物的繁殖物候基本重叠(李慢如和 张玲，2019)。但也有研究表明，寄主植物的物候与 桑寄生植物的物候没有相关性, 桑寄生植物的开花 和结果物候与它们的寄主并没有在时间上保持一 致，也没有相同的物候事件发生顺序(Yule \& Bronstein, 2018b)。由此可见，寄主植物在寄生植物 开花物候的进化过程中所产生的选择压力或许起 到了重要作用，但还尚未被充分地研究和证实。

\section{2 传粉者和种子散布者的影响}

(1)传粉者的影响：对于那些依赖动物传粉媒 介才能完成自身繁殖的桑寄生植物来说, 其物候可 能会受到这些传粉者的影响, 或受到与寄主植物竞 争这些媒介的限制(Yule \& Bronstein, 2018b)。印度 南部同域分布的6种桑寄生植物的繁殖物候就表现 
为种内花期与果期部分重叠, 种间花果期彼此错开 (Davidar, 1983b)。当共享传粉和散布种子的啄花鸟 属(Dicaeum)鸟类时, 植物个体选择暂时错开开花 时间, 减少种内开花时间的重叠, 从而达到异花授 粉和减少有限传粉者竞争的目的(Ashton et al, 1988; Wright \& Calderon, 1995)。

(2)种子散布者的影响：由于桑寄生植物的半 寄生性, 绝大多数种类都需要依靠体型较小的鸟类 和啮齿类动物等散布种子, 才有可能到达合适的寄 主完成定殖(Reid, 1989; Luo et al, 2016; Amico et al, 2017)。Aizen (2003)对巴塔哥尼亚西北部桑寄生科 植物Tristerix corymbosu的研究表明, 其种子散布者 Dromiciops australis在夏季最为活跃, 因此夏季成 熟的果实比春季和冬季有更大的散布机会。当互利 共生双方的物候在时间上存在物候错配 (phenological mismatch)时, 对于双方的代价都较大(Yule \& Bronstein, 2018b), 因此种子散布者特定的活动 阶段将有可能限制相应植物繁殖物候的进化(Aizen, 2003)。

\section{3 桑寄生植物个体特性的影响}

(1)个体相互作用的影响：对于寄生动物的研 究发现, 当两种寄生动物共享同一个寄主时, 要么 一种寄生有利于另一种寄生的定殖(比如, 削弱寄 主的抵抗力), 要么两种寄生会因共享寄主资源产 生直接的竞争，这将导致在同一个寄主上不同种类 的寄生个体物候发展不一致(Petney \& Andrews, 1998)。同理，同种寄生植物的不同个体在同一寄主 植物个体上也应存在一定的资源竞争，但根据现有 的对沙漠檞寄生的研究, 同一寄主个体上不同寄生 个体植物的始花期并没有显著的差异 (Yule \& Bronstein, 2018b)。对于这种靠昆虫传粉才能完成繁 殖的桑寄生植物来说, 在一定距离内保持花期同步 更能保障自身的繁殖成功 (Yule \& Bronstein, 2018a)。

(2)生物学特性的影响: 植物所具有的生物学 特性可以预测自身的开花时间(Du et al, 2015), 如 植物个体大小、高度等生物学特性可能会调节植物 的物候模式, 并表现在开花时间的高度变异 (Bolmgren \& Cowan, 2008; Jentsch et al, 2009; Davis et al, 2010; Sun \& Frelich, 2011)。花的数量、始花期 早晚、花期长短等都可以反映植物的大小(Schmitt,
1983)。如有关沙漠槲寄生植物P. californicum的研 究就表明, 在寄主植物Prosopis velutina上的沙漠槲 寄生个体较大，能产生更多的花粉和花蜜，盛花期 也要晚于在其他寄主植物种类上的盛花期(Yule \& Bronstein, 2018b)。但是该研究并没有揭示植物个体 大小对于植物的繁殖物候影响的原理。

\section{3 桑寄生植物繁殖物候的适应意义}

\section{1 桑寄生植物与传粉/种子散布者的相互作用}

分布于东非地区的桑寄生科植物 Phragmanthera dshallensis, 只有当取食的鸟类啄开成熟的花 冠管后才会产生花蜜(Gill \& Wolf, 1975), 这种机制 保障了乌在取食花蜜的时候可以携带大量新鲜的 花粉, 也确保了它们是第一个获取花蜜的拜访者 (Ladley et al, 1997)。同时，研究表明访花者对于不 是初开的花朵将会减少拜访(Davidar, 1983b), 所有 传粉者的受食阶段都与桑寄生植物开花物候阶段 相吻合(Quintana-Rodriguez et al, 2018)。在群落中多 种桑寄生植物季节性地开花和结果，彼此错开花期 和果期(Godschalk, 1983), 某些桑寄生植物为了吸 引一种食果鸟类拜访其花朵，会延迟果期的到来， 以增加自身的繁殖适合度(Davidar, 1983b)。

依靠鸟类和哺乳动物进行种子散布的桑寄生 果实大都含有质地粘稠的檞寄生素(viscin), 鸟类取 食以后通过排泄(defecation)或回吐(regurgitation)的 方式将黏性较强的种子放置在合适的寄主植物上 (Aukema, 2003; Lomascolo et al, 2010)。由于桑寄生 植物能与很多不同种类的寄主植物发生作用，尤其 当寄主植物与寄生植物共享种子散布者时, 它们之 间繁殖物候的重叠将对寄生植物种子的散布和定 殖有重要影响(Caraballo-Ortiz et al, 2017)。桑寄生植 物果期的延长有利于维持与其专性受食者的关系 (Larson, 1996), 从而维持与种子散布者的互利共生 (Tanaka \& Tokuda, 2017)。

\section{2 桑寄生植物与寄主植物的相互作用}

迄今, 虽然寄主植物与桑寄生植物之间繁殖物 候的关系还没有很好地被理解和研究，但是同步观 测并记录它们的物候被认为是一种分析寄主与桑 寄生植物相互作用的较容易的方式(Teixeira-Costa et al, 2017)。对于桑寄生植物来说，其物候有可能被 与其共享传粉者和种子散布者的寄主植物所限制 
(Yule \& Bronstein, 2018b)。当桑寄生植物与其寄主 植物的繁殖物候有部分重叠时, 被感染的寄主植物 个体的种子雨高于未被感染的同种植物个体(Candia et al, 2014); 同时, 不同寄主种类上寄生植物的 繁殖物候可以促进寄生植物自身种群的繁殖隔离, 从而加速桑寄生植物的物种分化(Yule \& Bronstein, 2018b)。

\section{4 总结与展望}

桑寄生植物不仅能与寄主植物、传粉者和种子 散布者发生直接作用，还能通过三者之间的关系间 接地影响林下调落物、微生物生物量、土壤湿度等, 从而形成地上-地下的有机整体(Watson, 2016)。因 此对桑寄生植物开展繁殖物候的研究有助于明确 它们为动物提供食物资源的时间范围, 为了解含桑 寄生植物群落中鸟类的多样性、林业防治中桑寄生 植物种群的管理以及自然生态系统中动植物关系 的研究都提供了科学依据(Press \& Phoenix, 2005; Teixeira-Costa et al, 2017)。

基于“桑寄生植物繁殖物候的异步是为了维持 与传粉者和种子散布者的互利共生关系”这一生态 学观点的研究表明, 该领域已从单一地描述繁殖物 候的表现类型到探究系统中寄主植物、传粉/种子散 布者以及桑寄生植物自身生物学特性等影响因素。 尽管关于寄主植物的种类和物候在影响桑寄生植 物繁殖物候的方面有不同的结论，但是随着分子生 物学技术的应用, 寄主植物对于桑寄生植物的影响 在未来的研究中可能会受到更多的重视。另外, 根 据前人研究的发展情况, 以下 3 个方面也需要进一 步加强研究:

(1)加强对桑寄生植物基础生物学背景的研究。 世界上约有 1,600 种桑寄生植物, 分布范围也较广 (Watson, 2011; Nickrent, 2011), 它们作为次级基础 物种(secondary foundation species)对于提高生物多 样性具有重要意义(Thomsen et al, 2018)。但关于桑 寄生植物繁殖生态学的研究还较少, 许多背景资料 不清楚, 这对进一步研究桑寄生植物与传粉者和种 子散布者之间的关系, 探讨生态系统功能十分不 利。Watson (2001)发表专著Mistletoes of Southern Australia, 对于澳洲南部分布的 45 种桑寄生植物的 生物学、生态学、文化价值和森林管理作出了详尽 的描述。经笔者对《中国植物志》第二十四卷(中国
科学院中国植物志编辑委员会，1988)和文献资料 (Zhang et al, 2018)的整理, 发现中国共分布桑寄生 植物3科(桑寄生科、槲寄生科和檀香科) 13属(桑寄 生属、槲寄生属和重寄生属等)80种和12变种(未发 表数据), 虽然对五荵寄生(D. pentandra) 的繁殖特 性进行研究发现该种植物不同的个体具有不同的 开花模式 ${ }^{1}$, 但整体而言, 桑寄生植物繁殖生态学 的研究还相对较少(夏博等, 2010; 随意, 2014 ${ }^{2}$; 严 光荣等, 2019), 因此我们需要加强对于该类植物群 的基础生物学背景的研究。

(2)实验验证桑寄生植物繁殖物候异步对维持 其与传粉/种子散布者互惠关系的作用。由于桑寄生 植物生活型的特殊性，目前还没有人为控制实验证 明桑寄生的繁殖物候异步性程度降低或者升高将 会产生怎样的生态后果。未来可以参照检验鸟类空 间记忆和搜索图像研究中对桑寄生植物的叶片和 植株的移除实验 ${ }^{3}$ ，通过人为去除那些较早开放的 花朵，使整个种群达到较高的开花同步性; 对果期 的验证亦可如此操作, 来检测桑寄生植物繁殖物候 的异步程度对“寄主植物-桑寄生植物-传粉/种子散 布者”系统中一方或多方的影响。

(3)综合多因素分析桑寄生植物繁殖物候的影 响因子。桑寄生植物的繁殖物候如何受到寄主植 物的种类以及物候、传粉者、种子散布者和自身 生物学特性的影响, 目前的研究工作还并不充分 (Teixeira-Costa et al, 2017; Yule \& Bronstein, 2018b)。因此可以考虑选取更多的寄主植物种类进 行深入研究, 探究桑寄生植物的繁殖物候与寄主植 物的关系; 除此之外, 还应加强非生物因素(温度、 光照和湿度等)对桑寄生植物和相应的寄主植物繁 殖物候影响的研究。

\section{参考文献}

Aizen MA (2003) Influences of animal pollination and seed dispersal on winter flowering in a temperate mistletoe. Ecology, 84, 2613-2627.

(1) 罗亚皇 (2012) 五荵寄生(Dendrophthoe pentandra)的繁殖特性及寄 生过程的生态适应性研究. 硕士学位论文, 中国科学院西双版纳热带植 物园, 云南预腊.

(2) 随意 (2014) 两种泛性寄生的繁殖特性和种子散布过程对其空间分 布格局的影响. 硕士学位论文, 中国科学院西双版纳热带植物园, 云南 预腊.

(3) Cook ME (2017) Spatial memory, search images and environmental cues: How do frugivores find ripe mistletoes fruits? Master dissertation, Faculty of Science, School of Life Sciences, University of Technology Sydney, Australia. 
Amico GC, Aizen MA (2000) Mistletoe seed dispersal by a marsupial. Nature, 408, 929-930.

Amico GC, Sasal Y, Vidal-Russell R, Aizen MA, Manuel MJ (2017) Consequences of disperser behaviour for seedling establishment of a mistletoe species. Austral Ecology, 42, 900-907.

Ashton PS, Givnish TJ, Appanah S (1988) Staggered flowering in the Dipterocarpaceae: New insights into floral induction and the evolution of mast fruiting in the aseasonal tropics. The American Naturalist, 132, 44-66.

Augspurger CK (1983) Phenology, flowering synchrony, and fruit set of six neotropical shrubs. Biotropica, 15, 257-267.

Aukema JE (2003) Vectors, viscin, and Viscaceae: Mistletoes as parasites, mutualists, and resources. Frontiers in Ecology and the Environment, 1, 212-219.

Bannister P, Strong GL (2001) Carbon and nitrogen isotope ratios, nitrogen content and heterotrophy in New Zealand mistletoes. Oecologia, 126, 10-20.

Barney CW, Hawksworth FG, Geils BW (1998) Hosts of Viscum album. European Journal of Forest Pathology, 28, 187-208.

Bolmgren K (1998) The use of synchronization measures in studies of plant reproductive phenology. Oikos, 82, 411-415.

Bolmgren K, Cowan PD (2008) Time-size tradeoffs: A phylogenetic comparative study of flowering time, plant height and seed mass in a north-temperate flora. Oikos, 117, 424-429.

Burkhardt F, Smith S (1990) The Correspondence of Charles Darwin. Cambridge University Press, Cambridge.

Candia AB, Medel R, Fonturbel FE (2014) Indirect positive effects of a parasitic plant on host pollination and seed dispersal. Oikos, 123, 1371-1376.

Caraballo-Ortiz MA, Gonzalez-Castro A, Yang S, dePamphilis CW, Carlo TA (2017) Dissecting the contributions of dispersal and host properties to the local abundance of a tropical mistletoe. Journal of Ecology, 105, 1657-1667.

Clark RM, Thompson R (2011) Estimation and comparison of flowering curves. Plant Ecology \& Diversity, 4, 189-200.

Craine JM, Wolkovich EM, Towne EG, Kembel SW (2012) Flowering phenology as a functional trait in a tallgrass prairie. New Phytologist, 193, 673-682.

Davidar P (1983a) Birds and neotropical mistletoes: Effects on seedling recruitment. Oecologia, 60, 271-273.

Davidar P (1983b) Similarity between flowers and fruits in some flowerpecker pollinated mistletoes. Biotropica, 15, 32-37.

Davis CC, Willis CG, Primack RB, Miller-Rushing AJ (2010) The importance of phylogeny to the study of phenological response to global climate change. Philosophical Transactions of the Royal Society B: Biological Sciences, 365, 3201-3213.
Delectis Flora Reipubicae Popularis Sinicae Agendae Academiae Sinicae Edita (1998) Flora Reipublicae Popularis Sinicae, Tomus 24. Science Press, Beijing. (in Chinese) [中 国科学院中国植物志编辑委员会 (1998) 中国植物志(第 二十四卷). 科学出版社, 北京.]

Du YJ, Mao LF, Queenborough SA, Freckleton RP, Chen B, Ma KP (2015) Phylogenetic constraints and trait correlates of flowering phenology in the angiosperm flora of China. Global Ecology and Biogeography, 24, 928-938.

Elzinga JA, Atlan A, Biere A, Gigord L, Weis AE, Bernasconi G (2007) Time after time: Flowering phenology and biotic interactions. Trends in Ecology \& Evolution, 22, 432-439.

Feehan J (1985) Explosive flower opening in ornithophily: A study of pollination mechanisms in some Central African Loranthaceae. Botanical Journal of the Linnean Society, 90, 129-144.

Gill FB, Wolf LL (1975) Foraging strategies and energetics of East African sunbirds at mistletoe flowers. The American Naturalist, 109, 491-510.

Godschalk SKB (1983) The reproductive phenology of three mistletoe species in the Loskop Dam Nature Reserve, South Africa. South African Journal of Botany, 2, 9-14.

Gorchov DL (1988) Does asynchronous fruit ripening avoid satiation of seed dispersers?: A field test. Ecology, 69, 1545-1551.

Jentsch A, Kreyling J, Boettcher-Treschkow J, Beierkuhnlein C (2009) Beyond gradual warming: Extreme weather events alter flower phenology of European grassland and heath species. Global Change Biology, 15, 837-849.

Ladley JJ, Kelly D (1995) Explosive New Zealand mistletoe. Nature, 378, 766.

Ladley JJ, Kelly D, Robertson AW (1997) Explosive flowering, nectar production, breeding systems, and pollinators of New Zealand mistletoes (Loranthaceae). New Zealand Journal of Botany, 35, 345-360.

Larson DL (1996) Seed dispersal by specialist versus generalist foragers: The plant's perspective. Oikos, 76, 113-120.

Li MR, Zhang L (2019) Reproductive phenological characteristics and impact of Macrosolen cochinchinensis in Xishuangbanna. Guihaia, 39, 1252-1260. (in Chinese with English abstract) [李慢如, 张玲 (2019) 西双版纳地区鞘 花的繁殖物候及影响因素. 广西植物, 39, 1252-1260.]

Liu B, Chi TL, Barrett RL, Nickrent DL, Chen ZD, Lu LM, Vidal-Russell R (2018) Historical biogeography of Loranthaceae (Santalales): Diversification agrees with emergence of tropical forests and radiation of songbirds. Molecular Phylogenetics and Evolution, 124, 199-212.

Lomascolo SB, Levey DJ, Kimball RT, Bolker BM, Alborn HT (2010) Dispersers shape fruit diversity in Ficus (Moraceae). Proceedings of the National Academy of Sciences, USA, 107, 14668-14672.

Luo YH, Sui Y, Gan JM, Zhang L (2016) Host compatibility interacts with seed dispersal to determine small-scale 
distribution of a mistletoe in Xishuangbanna, Southwest China. Journal of Plant Ecology, 9, 77-86.

Mathiasen RL, Nickrent DL, Shaw DC, Watson DM (2008) Mistletoes: Pathology, systematics, ecology, and management. Plant Disease, 92, 988-1006.

Napier KR, Mather SH, McWhorter TJ, Fleming Patricia A (2014) Do bird species richness and community structure vary with mistletoe flowering and fruiting in Western Australia? Emu, 114, 13-22.

Newstrom LE, Frankie GW, Baker HG (1994) A new classification for plant phenology based on flowering patterns in lowland tropical rain-forest trees at La selva, Costa-rica. Biotropica, 26, 141-159.

Nickrent DL (2002) Mistletoe phylogenetics: Current relationships gained from analysis of DNA sequences. In: Proceedings of the Western International Forest Disease Work Conference, p. 253. Waikoloa, Hawai'i.

Nickrent DL (2011) Santalales (including mistletoes). In: Encyclopedia of Life Sciences (ELS). John Wiley \& Sons, Chichester.

Overton JM (1997) Host specialization and partial reproductive isolation in desert mistletoe (Phoradendron californicum). Southwestern Naturalist, 42, 201-209.

Petney TN, Andrews RH (1998) Multiparasite communities in animals and humans: Frequency, structure and pathogenic significance. International Journal for Parasitology, 28, 377-393.

Pettersson MW (1994) Large plant size counteracts early seed predation during the extended flowering season of a Silene uniflora (Caryophyllaceae) population. Ecography, 17, 264-271.

Press MC, Phoenix GK (2005) Impacts of parasitic plants on natural communities. New Phytologist, 166, 737-751.

Quintana-Rodriguez E, Gamaliel RA, Ramirez-Chavez E, Molina-Torres J, Camacho-Coronel X, Esparza-Claudio J, Heil M, Orona-Tamayo D (2018) Biochemical traits in the flower lifetime of a Mexican mistletoe parasitizing mesquite biomass. Frontiers in Plant Science, 9, 1031.

Rathcke B, Lacey EP (1985) Phenological patterns of terrestrial plants. Annual Review of Ecology and Systematics, 16, 179-214.

Reid N (1989) Dispersal of mistletoes by honeyeaters and flowerpeckers: Components of seed dispersal quality. Ecology, 70, 137-145.

Reid N (1991) Coevolution of mistletoes and frugivorous birds. Australian Journal of Ecology, 16, 457-469.

SanMartin-Gajardo I, Morellato LPC (2003) Inter and intraspecific variation on reproductive phenology of the Brazilian Atlantic forest Rubiaceae: Ecology and phylogenetic constraints. Revista de Biologia Tropical, 51, 691-698.

Schmitt J (1983) Individual flowering phenology, plant size, and reproductive success in Linanthus androsaceus, a Cali- fornia annual. Oecologia, 59, 135-140.

Shen H, Ye W, Hong L, Huang H, Wang Z, Deng X, Yang Q, $\mathrm{Xu} \mathrm{Z}$ (2006) Progress in parasitic plant biology: Host selection and nutrient transfer. Plant Biology, 8, 175-185.

Sun SC, Frelich LE (2011) Flowering phenology and height growth pattern are associated with maximum plant height, relative growth rate and stem tissue mass density in herbaceous grassland species. Journal of Ecology, 99, 991-1000.

Tanaka K, Tokuda M (2017) Phenological specialisation of two ant-dispersed sedges in relation to requirements for qualitative and quantitative dispersal effectiveness. Ecological Research, 32, 677-684.

Teixeira-Costa L, Coelho FM, Ceccantini GCT (2017) Comparative phenology of mistletoes shows effect of different host species and temporal niche partitioning. Botany, 95, 271-282.

Thompson JN, Willson MF (1979) Evolution of temperate fruit-bird interactions: Phenological strategies. Evolution, 33, 973-982.

Thomsen MS, Altieri AH, Angelini C, Bishop MJ, Gribben PE, Lear G, He Q, Schiel DR, Silliman BR, South PM, Watson DM, Wernberg T, Zotz G (2018) Secondary foundation species enhance biodiversity. Nature Ecology \& Evolution, 2, 634-639.

Twyford AD (2018) Parasitic plants. Current Biology, 28, R857-R859.

Vaknin Y, Tov YY, Eisikowitch D (1996) Flowering seasonality and flower characteristics of Loranthus acaciae Zucc. (Loranthaceae): Implications for advertisement and birdpollination. Sexual Plant Reproduction, 9, 279-285.

Wang LX, Kgope B, D’Odorico P, Macko SA (2008) Carbon and nitrogen parasitism by a xylem-tapping mistletoe (Tapinanthus oleifolius) along the Kalahari Transect: A stable isotope study. African Journal of Ecology, 46, 540-546.

Wang XN, Zhang L (2017) Species diversity and distribution of mistletoes and hosts in four different habitats in Xishuangbanna, Southwest China. Journal of Yunnan University (Natural Science), 39, 701-711. (in Chinese with English abstract) [王煊妮, 张玲 (2017) 西双版纳4种生境 下的桑寄生与寄主植物多样性及分布特点. 云南大学学 报(自然科学版), 39, 701-711.]

Watson DM (2001) Mistletoe-A keystone resource in forests and woodlands worldwide. Annual Review of Ecology and Systematics, 32, 219-249.

Watson DM (2011) Mistletoes of Southern Australia. CSIRO Publishing, Collingwood.

Watson DM (2016) Fleshing out facilitation-reframing interaction networks beyond top-down versus bottom-up. New Phytologist, 211, 803-808.

Watson DM, Herring M (2012) Mistletoe as a keystone resource: An experimental test. Proceedings of the Royal Society B: Biological Sciences, 279, 3853-3860. 
Wright JW, Meagher TR (2003) Pollination and seed predation drive flowering phenology in Silene latifolia (Caryophyllaceae). Ecology, 84, 2062-2073.

Wright SJ, Calderon O (1995) Phylogenetic patterns among tropical flowering phenologies. Journal of Ecology, 83, 937-948.

Xia B, Tian CM, Luo YQ, Zhao FY, Ma JH, Wang GC, Han FZ (2010) Flowering characteristics and chemical control of the buds of Arceuthobium sichuanense. Scientia Silvae Sinicae, 46(4), 98-102. (in Chinese with English abstract) [夏博, 田呈明, 骆有庆, 赵丰钰, 马建海, 王国仓, 韩富 忠 (2010) 云杉矮槲寄生开花特性及化学防控. 林业科 学, 46(4), 98-102.]

Yan GR, Yang YQ, Tang AJ (2019) Investigating seed characteristics and parasitic relation establishment of Scurrula parasitica var. graciliflora characterized by prior selfing. Acta Ecologica Sinica, 39, 1019-1029. (in Chinese with English abstract) [严光荣, 杨永清, 唐安军 (2019) 前自交型小红花种子特性及寄生关系的建立. 生态学报, 39, 1019-1029.]

Yule KM, Bronstein JL (2018a) Infrapopulation size and mate availability influence reproductive success of a parasitic plant. Journal of Ecology, 106, 1972-1982.

Yule KM, Bronstein JL (2018b) Reproductive ecology of a parasitic plant differs by host species: Vector interactions and the maintenance of host races. Oecologia, 186, 471-482.

Yule KM, Koop JAH, Alexandre NM, Johnston LR, Whiteman NK (2016) Population structure of a vector-borne plant parasite. Molecular Ecology, 25, 3332-3343.

Zhang GF, Li Q, Sun SC (2018) Diversity and distribution of parasitic angiosperms in China. Ecology and Evolution, 8, 4378-4386.

(责任编委：张大勇 责任编辑：时意专) 